\title{
3D-PIV Measurement for EHD Flow of Spiked Tubular Electrode Corona Discharge in Wide Electrostatic Precipitator
}

\author{
Dongjie Yan ${ }^{1,2}$, Ziang Zhang ${ }^{1,2}$, Zhenyang $\mathrm{Li}^{2}$, Ya Yu ${ }^{1,2}$, Hao Gong ${ }^{1,2}$, Xueming Huang ${ }^{2}$ \\ ${ }^{1}$ School of Environmental and Municipal Engineering, Xi'an University of Architectural Science and Technology, Yanta \\ Anvenue, No.13, 710055,Xi'an, China, ziang mengjie@163.com \\ ${ }^{2}$ Shaanxi Key Laboratory of Environmental Engineering, Xi' an University of Architecture and Technology, Yanta Anvenue, \\ No.13, 710055, Xi'an, China
}

\begin{abstract}
The electrohydrodynamic (EHD) flow induced by a corona discharge has an important influence on the movement and collection of fine particles in an electrostatic precipitator. In this paper, three-dimensional particle image velocimetry (3D-PIV) is used to investigate the impact of different primary flow velocities and applied voltage on diffusion and transport of the spiked tubular electrode corona discharge EHD flow in a wide type electrostatic precipitator. In order to measure the flow characteristics of different positions of a spiked tubular electrode, the PIV measurements are carried out in several cross-sectional planes along the ESP duct. From 2D flow streamlines, in plane 1 (where the tip of the spike is oriented in the direction of primary flow), the velocity of the counter-clockwise vortex caused by the EHD flow near the plate decreases as the primary flow velocity increases. However, in plane 3 (where the tip direction is opposite to the primary flow), two vortices rotate adversely, and the flow velocity of the clockwise vortex near the plate increases as the primary flow velocity increases. Flow velocity increasing near the plate makes the particles deposited on the plate more easily to be re-entrained. It can be found in the three-dimensional analysis of the flow field that there are mainly "ascending vortex" and downward tip jet in the three observation planes. There is a discrepancy (in terms of distribution region and the magnitude of velocity) between the three-dimensional characteristics of these vortices and tip jets in the different cross-sectional planes.
\end{abstract}

Keywords: Electrostatic precipitator; 3D-PIV; EHD flow; flow characteristics; spike tubular electrode.

\section{INTRODUCTION}

An electrostatic precipitator is an efficient filtration device for particulate matter, and its total mass collection efficiency of particulate matter can reach over $99 \%$. However, its collection efficiency of submicron particles (of diameter range of $0.1-1 \mu \mathrm{m}$ ) is much lower [1], resulting in a high proportion of submicron particles in the outlet dust. Submicron particles containing hazardous trace elements easily penetrate into the respiratory system causing adverse effects to human body [2]-[3]. For these reasons, many governments have emission standards set for particulate matter.

The collection of fine particles in an electrostatic precipitator is affected by the comprehensive function of the electric field and the fluid field. The electrohydrodynamic (EHD) flow induced by corona discharge is formed by the coupling of ionic wind and mean-flow (also known as the primary flow) in the collection channel [4]-[6], which can disrupt flow patterns and cause complicated turbulences [7][8]. The existence of such non-laminar flow structure will hamper the particle collection and complicate the removal mechanism of the fine particles [9]. Therefore, it is important to study the motion characteristics of gas flow and particles in this process of corona discharge.

The research of EHD flow characteristics in electrostatic precipitators can be traced back to the last century. In recent years, the particle image velocimetry (PIV) has become a powerful tool for measuring the EHD flow. Podlinski et al. studied the EHD characteristics of wire-plate [10]-[11] and spike-plate [12]-[14] electrostatic precipitators under positive and negative corona discharge by PIV. Mizeraczyk et al. studied the discharge EHD flow of a needle-plate [15] in finite-volume chamber. Miyashita et al. used 2D-PIV to study particle behavior under the influence of ion wind in a hole-type electrostatic precipitator [16]. The above investigation used a narrow electrostatic precipitator with plate distance of $30 \mathrm{~mm}-200 \mathrm{~mm}$. However, in practical industrial applications, the distance between plate electrodes in electrostatic precipitators is greater than or equal to $400 \mathrm{~mm}$. Hence, a research group at Zhejiang University studied EHD in a wide-type electrostatic precipitator [17][18]. However, their research method was limited to the use of 2D-PIV technology. Up to now, there are few studies of wide-type electrostatic precipitators using 3D-PIV technology. 
The spiked tubular electrode is widely used in particle collection owing to its high discharge intensity and low corona onset voltage. However, corona discharge of this electrode generates a strong EHD flow, interfering with the primary flow and the particle movement. In this experiment, the 3D-PIV was used to study the EHD flow of the spiked tubular electrode corona discharge with a $400 \mathrm{~mm}$ plate electrode distance. The paper focuses on the vortex characteristics under the influence of EHD and its influence on the flow field near the plate electrode. Additionally, the three-dimensional characteristics of EHD in the flow field are investigated. The content of this paper provides a reference for visualization research of air-flow field and particle movement in an electrostatic precipitator.

\section{SUBJECT \& METHODS}

\section{A. Experimental setup}

The apparatus used in this experiment is shown in Fig.1. It mainly consists of an electrostatic precipitator model, a high voltage power supply device, a fog machine, a fan, and a standard 3D-PIV system.

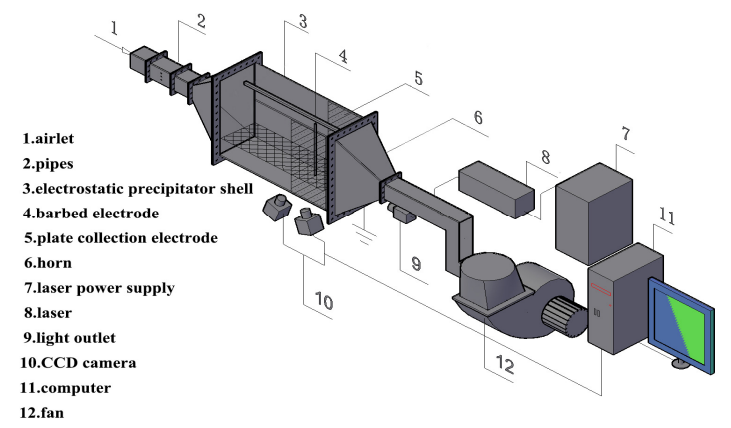

Fig.1. Experimental device diagram.

The 3D-PIV, also known as stereoscopic PIV, can be divided into 2D-3CPIV and 3D-3CPIV. The test system used in this experiment was 2D-3CPIV. Compared to 2DPIV, which uses only one camera used in 2D-PIV, this system used the extract particle images. By using two different viewing angles, one can obtain projections of the velocity vector in two planes. It is necessary to determine the active position and orientation of both cameras with respect to the orientation of the light sheet position through the process of calibration (the camera viewing directions are shown in Fig.2.). Once the arrangement of both cameras is known, one can extract velocity components in the three speed directions from the two projections.

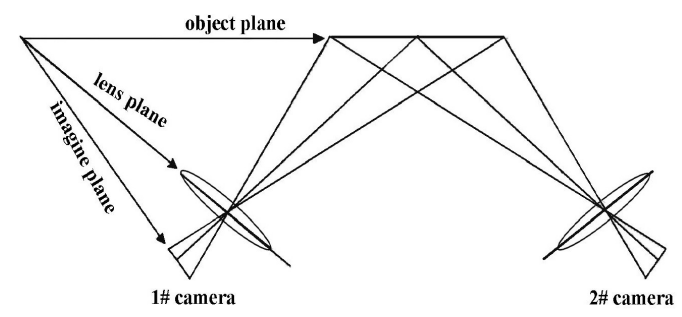

Fig.2. Camera arrangement of 2D-3CPIV.
The PIV includes a laser, a CCD camera, and data processing equipment. A double pulse $\mathrm{Nd}$ :YAG laser provides the light source. The laser emits light through the light guide arm, which converts the laser into a sheet light of $0.3 \sim 10 \mathrm{~mm}$ thickness. The camera is an Imager LX 29M, with resolution of $6600 \times 4400$ and pixel size of $5.5 \mu \mathrm{m}$ $\times 5.5 \mu \mathrm{m}$. The analysis software is DaVis software, and it has an image analysis processing function, which can analyze the flow field in detail and obtain relevant flow field data such as average velocity and velocity distribution.

The electrostatic precipitator (ESP) model is a parallel hexahedron made of acrylic material. It has inlet and outlet pipes, inlet and outlet horns, precipitator shell, and power supply. Baffle and air distribution plates are installed in the inlet and outlet horn to homogenize the air flow of the discharge region. The electrical electrode set consists of a discharge electrode (spiked tubular electrode) and collection electrodes (C-type plate electrodes); this electrode configuration is described in detail later.

The power source used in the experiment is the EHC-II DC power supply, which is a high-frequency and highvoltage power supply specially used for ESP. The negative DC voltage applied to the spiked tubular electrode was up to $75 \mathrm{kV}$ and the discharge current was up to $400 \mu \mathrm{A}$. Compared with ordinary DC power supply, high frequency power supply has the advantages of small voltage fluctuation and stable current, being beneficial to the stable observation of the flow field. The voltage and current (realtime and average) of high-voltage discharge are checked by a hand-operated device.

\section{B. Experiment and methods}

A smoke seeded with tracer particles was introduced into the gas inlet. The tracer particles used in the experiment were oil fog (of average particle diameter of $1.5 \mu \mathrm{m}$ ) emitted from a fog machine. Wang et al. [19] used the same kind of tracer particles to study EHD flow transition in ESP. When the laser system is turned on, the laser beam is injected from one side of the electrostatic precipitator through the light guide arm. The laser light sheet is perpendicular to the discharge electrode, and the collection electrode passes through the electric field area of the electrostatic precipitator. Then the tracer particles encounter the laser sheet to form a scattered optical field. At the same time, the flow field in the observation area is recorded by the camera and the images are stored on the computer.

The particles in the flow are illuminated twice, with a small-time separation in between. The displacement of particles in the time between the laser pulses is recorded as either a single image exposed twice or as a pair of two single exposure images. Using the cross-correlation calculation Davis software, the trajectories of particles in the two photos are analyzed, and the $2 \mathrm{D}$ and $3 \mathrm{D}$ flow field information is obtained by combining the calculation results of each particle cluster. The calculated results are postprocessed by Tecplot software and displayed in the form of a streamline diagram. The two-dimensional characteristics of the flow field in the ESP are studied, based on the twodimensional images study. By combining 2D and 3D images, 
the three-dimensional characteristics of particle movement in the dust collector are investigated, and the z-direction velocity and its influence on the flow field are also investigated.

\section{Observation area}

The observation position is shown in Fig.2. As can be seen, there were two observation areas (area A and area B) and three observation planes (plane 1, plane 2, and plane 3) in the experiment. The spiked tubular electrode is at the center of the discharged region, and the image range is about $230 \mathrm{~mm} \times 350 \mathrm{~mm}$. In area $\mathrm{B}$, the observation area is between the tubular electrode and the plate electrode; the imaging area is $200 \mathrm{~mm} \times 350 \mathrm{~mm}$. The above two observation areas can completely record the data of the flow field of the collection duct. Furthermore, the three observation planes were selected to investigate the influence of the tip position on the flow field. In plane 1, the spike tip is placed in the opposite direction to the primary airflow. In plane 3, the tip of the spike is placed in the same direction of the primary airflow. Plane 2 passed in-between the two tips mentioned above. The electrode configuration is also shown in Fig.2. The discharge electrode is a modified spiked tubular electrode (a nested tubular structure: diameter $20 \mathrm{~mm}, 750 \mathrm{~mm}$ long, and $100 \mathrm{~mm}$ distance between the midpoint of the two adjacent tips). The collection electrodes are three sets of $480 \mathrm{~mm}$ wide stainless-steel plates placed symmetrically (grounded). The distance between plates is $400 \mathrm{~mm}$, and the windbreak region is at both ends of the plate to reduce the flow velocity near the plate. Compared with previous studies, this electrode configuration is closer to the actual industrial application.
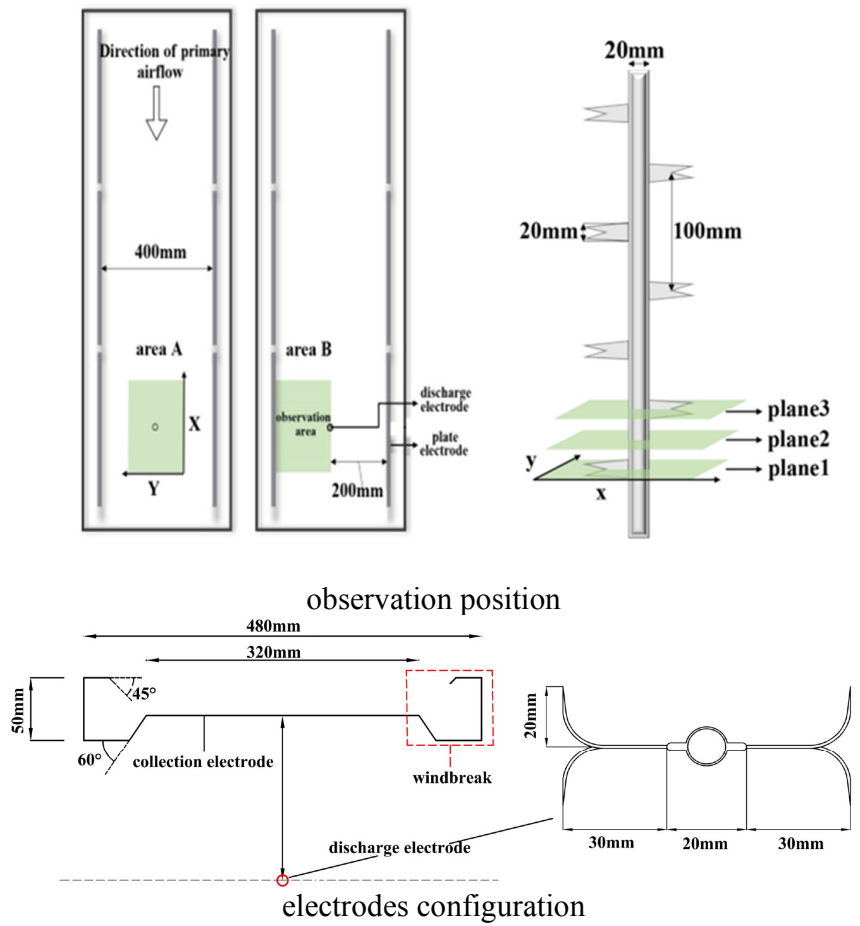

Fig.3. Observation position and electrodes configuration.

\section{RESULTS AND ANALYSIS}

\section{A. Research on symmetry of wide flow field}

The image width of the PIV system is $230 \mathrm{~mm}$, which cannot reach the plate electrode distance of $400 \mathrm{~mm}$. Due to this limitation, the flow field of the whole collection duct of electrostatic precipitator cannot be observed in a single position measurement. However, in this experiment, it can be found that the flow field is symmetrical in the duct of the electrostatic precipitator. Fig.4. is the flow streamline diagram of area A. The $\mathrm{x}$-axis in this figure is parallel to the plate electrodes and its negative direction is directed to the primary flow, the $y$-axis is perpendicular to the plate electrodes, corresponding to the $\mathrm{x}$ and $\mathrm{y}$ directions in Fig.2. When no voltage is applied, the flow field presents regular horizontal flow (see Fig.4.a)). When $45 \mathrm{kV}$ voltage is applied, it can be observed that the streamline near the spike bends and forms a vortex. When the tip of the spike is in the direction of the primary flow (see Fig.4.b)) and in the opposite direction of the primary flow (see Fig.4.c)), vortices are produced on both sides of the spike tip and the flow pattern is symmetrical around the spiked tubular electrode. The tilted data-free area in the picture caused by the spiked tubular electrode blocks part of the laser light during the shooting process. In order to avoid errors in the calculation process, this part of the data is ignored.

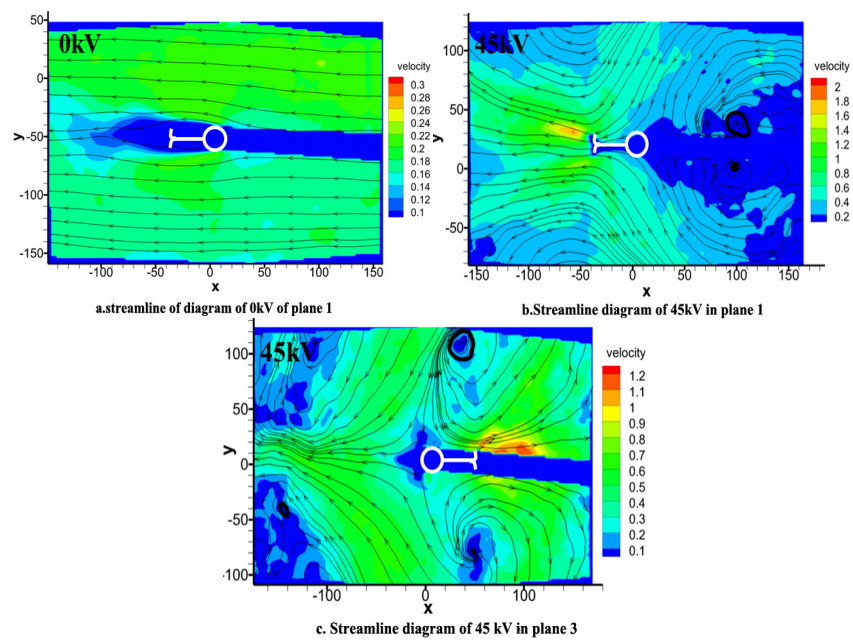

Fig.4. Symmetrical streamline diagram in area A (primary flow velocity of $0.2 \mathrm{~m} / \mathrm{s}$ ).

There is about $110 \mathrm{~mm}$ overlap between area A and area B. The splicing diagram is shown in Fig.5.a) and Fig.5.b) at the voltage of $45 \mathrm{kV}$ and $70 \mathrm{kV}$, respectively. It can be noticed that when the flow fields of area A and area B are spliced, the streamline and the velocity distribution have continuity in plane 1 , and plane 3 is basically the same. The comparison of the two pieces of experimental data also shows that the flow field in the wide electrostatic precipitator is symmetrical around the section of spiked tubular electrode, therefore, it can be simplified to study the characteristics of the flow field in the channel between plate and wire electrode. The following study area is all flow field characteristics of the area $\mathrm{B}$ region. 


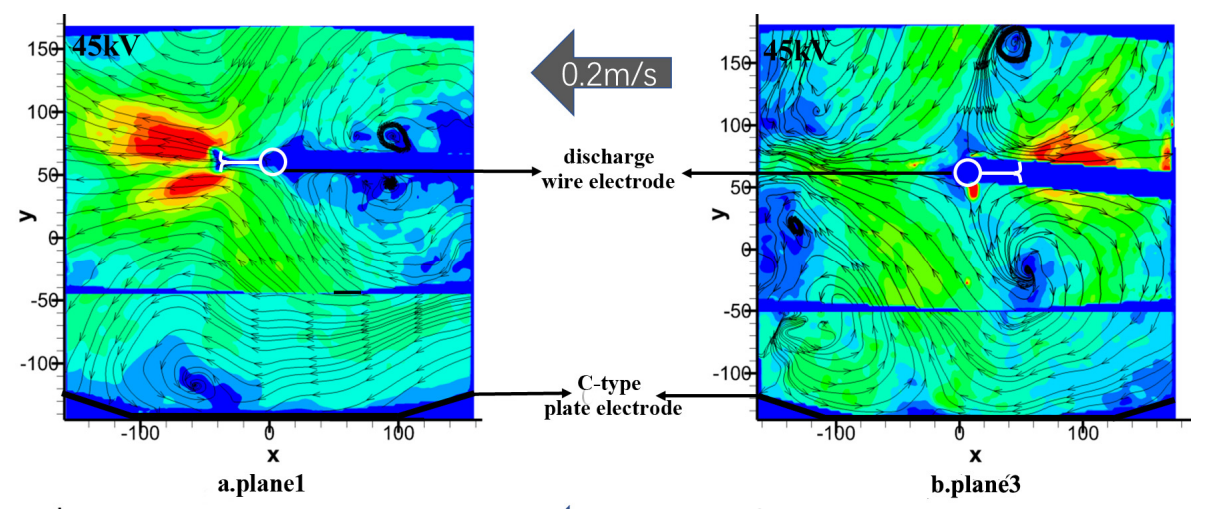

Fig.5.a) Splicing streamlines diagram at $45 \mathrm{kV}$.

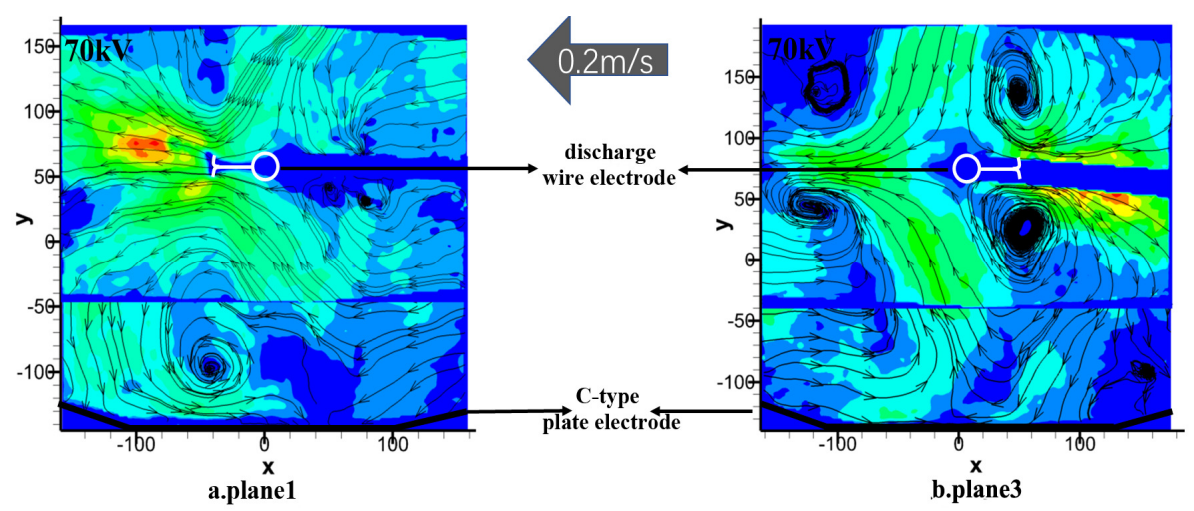

Fig.5.b) Splicing streamlines diagram at $70 \mathrm{kV}$.

\section{B. Flow field measurement at plane 1}

The streamline diagram in the duct of the electrostatic precipitator applied different voltage and primary flow velocity in plane 1, as shown in Fig.6. When the primary flow velocity $\mathrm{U}_{0}$ is $0.2 \mathrm{~m} / \mathrm{s}$, and the applied voltage is $45 \mathrm{kV}$, the particles in the electrostatic precipitator deviate from the primary flow and begin to move toward the plate, resulting in a motion of an anti-clockwise rotation vortex. Also, a high-speed region is formed around the vortex, and a low velocity region is formed in the center of the vortex. The vortex morphology is similar to that in a study by Podlinski et al. [20]. At this time, the airflow velocity around the vortex (near the plate) is $0.17 \mathrm{~m} / \mathrm{s}$. With a voltage increase, the overall velocity of the counter-clockwise vortex also increases. When $\mathrm{U}_{0}$ is $0.2 \mathrm{~m} / \mathrm{s}$, and the applied voltage is $70 \mathrm{kV}$, the maximum jet velocity at the tip of the spike reaches $1.4 \mathrm{~m} / \mathrm{s}$ (7 times of the primary flow velocity) and the air velocity near the plate electrode reaches $0.85 \mathrm{~m} / \mathrm{s}$ (more than 4 times of the primary flow velocity). Such a phenomenon may make it easier for particles to move towards the plate and be collected better. It is due to the apparent low-speed region near the bending region of the Cshaped plate that particles may be better deposited on the plate without being re-entrained into the dust removal channel by a high-speed jet.
When $\mathrm{U}_{0}$ is $0.4 \mathrm{~m} / \mathrm{s}$, although the airflow deflection tends to be formed at $45 \mathrm{kV}$ and $50 \mathrm{kV}$, it is difficult to form a vortex near the plate electrode as was with $0.2 \mathrm{~m} / \mathrm{s}$. It was not until $60 \mathrm{kV}$ that a visible vortex was formed near the plate, which indicated that the voltage needed to form the vortex increased. This is due to the fact that a more significant mainstream velocity will reduce the effect of the EHD flow. At $70 \mathrm{kV}$, the jet velocity generated at the tip of the spike wire is $1.7 \mathrm{~m} / \mathrm{s}$, and the air velocity near the electrode plate is $0.76 \mathrm{~m} / \mathrm{s}$.

When $\mathrm{U}_{0}$ reaches $0.6 \mathrm{~m} / \mathrm{s}$, only a slight deflection can be seen at $45 \mathrm{kV}$ caused by the EHD flow. Along the y-axis direction, from the spiked tubular electrode to the plate, the air flow velocity gradually decreases. At $70 \mathrm{kV}$, the EHD flow acts to cause the vortex motion of the airflow in the duct of electrostatic precipitator and the flow velocity near the plate is $0.7 \mathrm{~m} / \mathrm{s}$. It can be found that as the flow velocity increases, the velocity near the plate gradually decreases. The reason for this phenomenon is that the rotation direction of the vortex is counter-clockwise in plane 1, which is opposite to the primary flow direction near the plate electrode. Therefore, the increase in the primary flow velocity hindered the vortex flow near the re-entrainment in the bending region of the C-type plate, resulting in a decrease in the flow velocity. This low velocity region is conducive to prevent re-entrainment and improve the collection efficiency. 


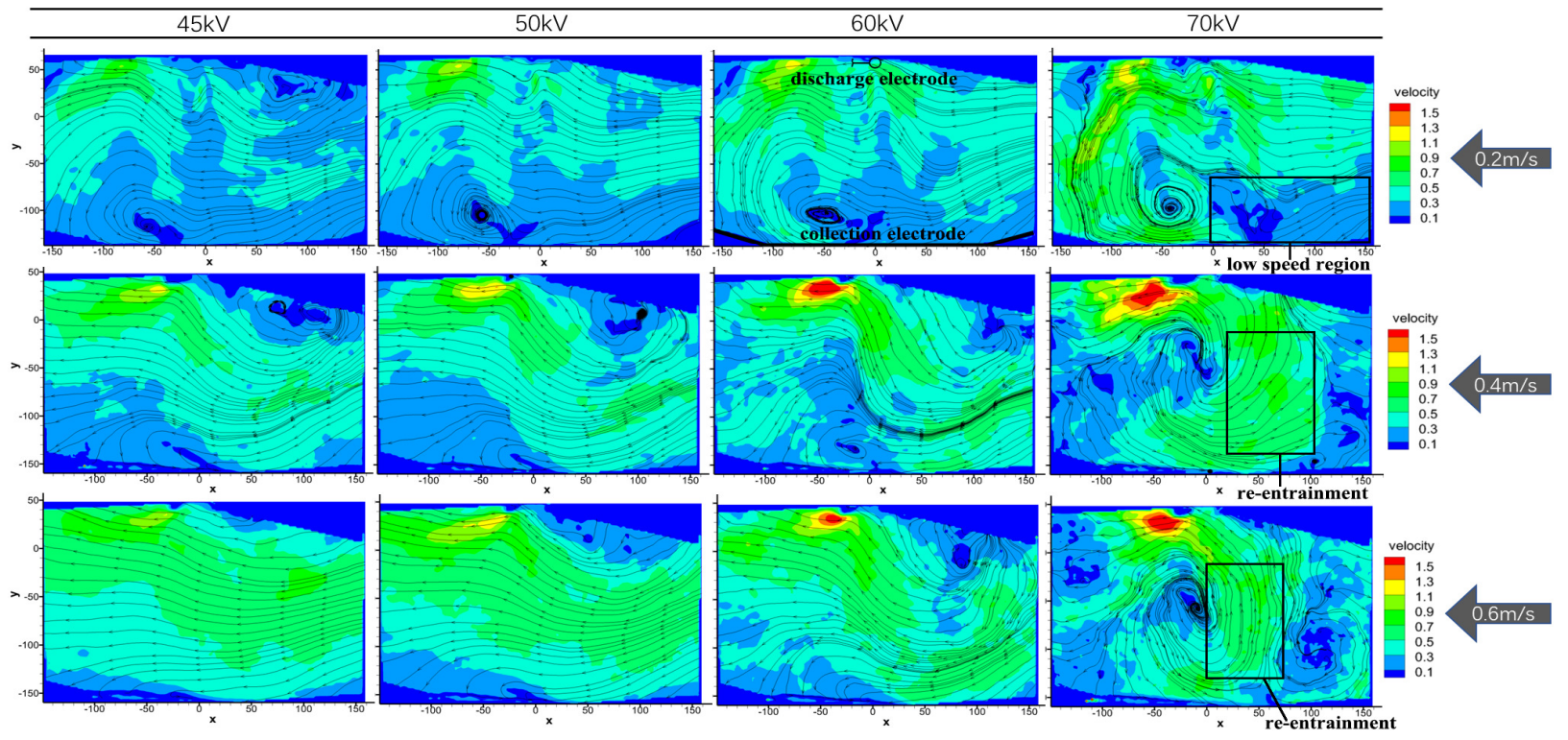

Fig.6. Streamlines with different primary flow velocity and voltages in plane 1 .

\section{Flow field analysis under plane 3}

The streamlines with different primary flow velocity and applied voltages in plane 3 are shown in Fig.7. In this plane, the tip direction is in the opposite direction to the primary flow. When the primary flow velocity (in plane 3 ) is $0.2 \mathrm{~m} / \mathrm{s}$, a clockwise vortex is formed at the upstream of the collection duct. Meanwhile, due to the primary flow and the compression of the collection duct, another counterclockwise vortex is formed in the downstream of the duct. It can be observed that the vortex can be formed at $45 \mathrm{kV}$ $\left(\mathrm{U}_{0}=0.2 \mathrm{~m} / \mathrm{s}\right)$. When the voltage rises to $70 \mathrm{kV}$, the maximum velocity of the jet flow reaches $1.7 \mathrm{~m} / \mathrm{s}$, and the flow velocity near the plate is about 0.45 .

With an increase of the primary flow velocity, the counterclockwise vortex almost disappears in the downstream of the duct at low voltage. However, the clockwise vortex makes the flow velocity near the plate increase. As can be seen, when the voltage is $70 \mathrm{kV}\left(\mathrm{U}_{0}=0.4 \mathrm{~m} / \mathrm{s}\right)$, the flow velocity near the plate reaches $0.7 \mathrm{~m} / \mathrm{s}$. At the same voltage, as the flow velocity increases to $0.6 \mathrm{~m} / \mathrm{s}$, the flow velocity near the plate reaches $0.75 \mathrm{~m} / \mathrm{s}$. The reason for this phenomenon is that primary flow is in the same direction of the vortex flow near the plate. Hence, increasing primary flow promotes the velocity in the vortex area near the plate. Therefore, under the influence of the clockwise vortex, the possibility of re-entrainment of particles near the plates increases.

The flow velocity variation near the plate electrode at different primary flow velocity and voltages in plane 1 and plane 3 is shown in Fig.8.

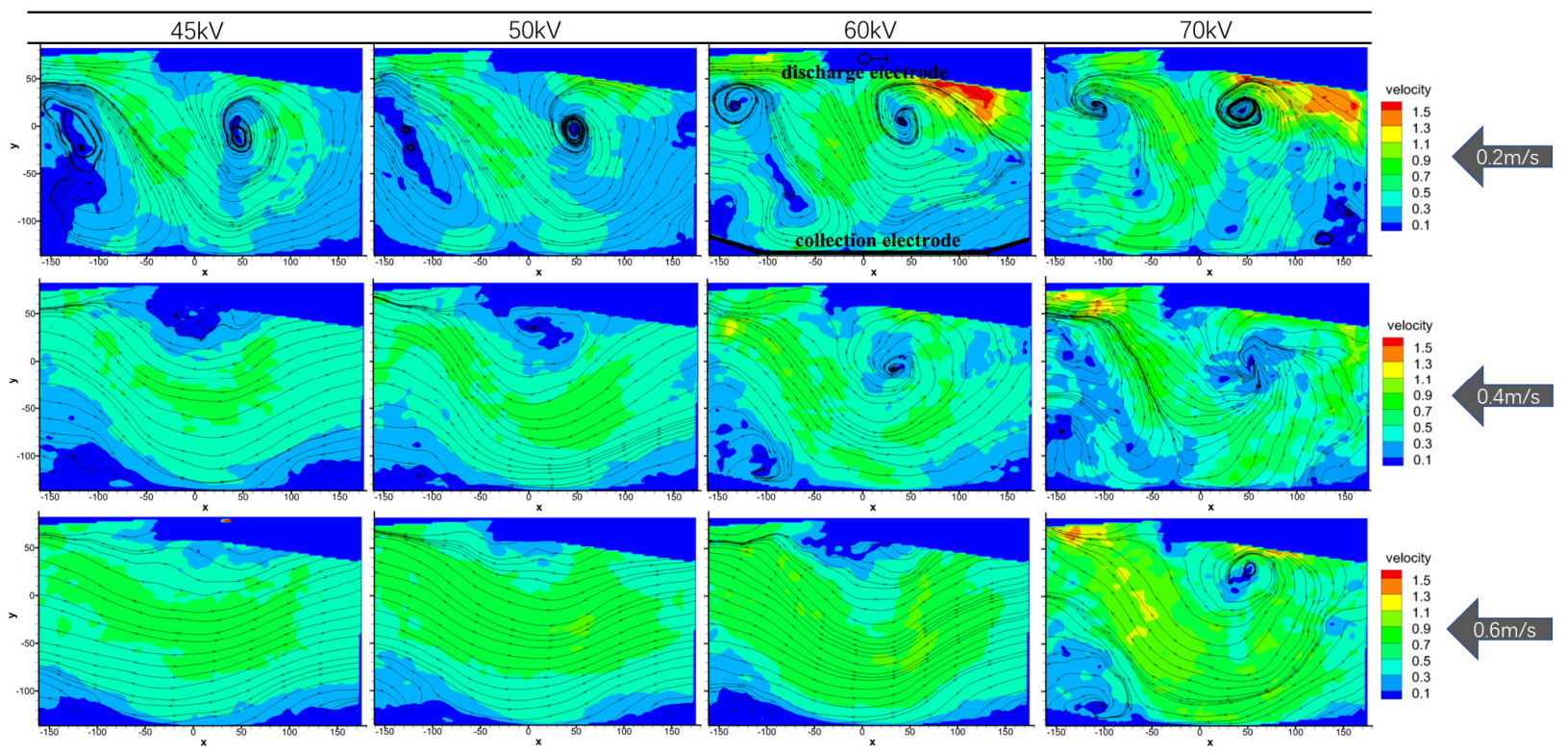

Fig.7. Streamlines with different primary flow velocity and voltages in plane 3 . 


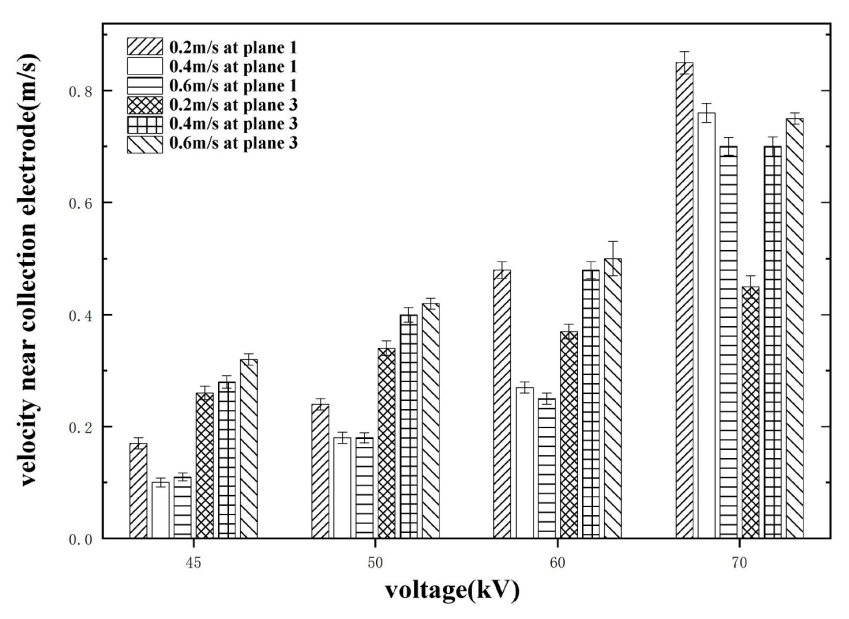

Fig.8. The flow velocity variation near the plate electrode influenced by EHD flow with different voltage and plane.
From this figure, we can observe that there is a positive correlation between velocity near the plate electrode and the magnitude of the applied voltage. At the same voltage, the flow velocity near the plate electrode decreases with an increase in primary flow velocity in plane 1. On the contrary, velocity near the plate electrode increases with an increase in the primary flow velocity in plane 3 .

\section{Flow field analysis in plane 2}

The flow pattern in plane 2 (located in the middle between plane 1 and plane 3) is shown in Fig.9. The vortex morphology, visible in the streamline diagram, is similar to that of plane 3. Nevertheless, the flow field characteristics are relatively confusing. When $\mathrm{U}_{0}$ is $0.2 \mathrm{~m} / \mathrm{s}$, the EHD flow induces two vortices. However, the velocities of these two vortices are smaller than those of plane 3. As the mainstream speed increases, the vortex near the plates disappears, and the air distribution becomes more uniform.

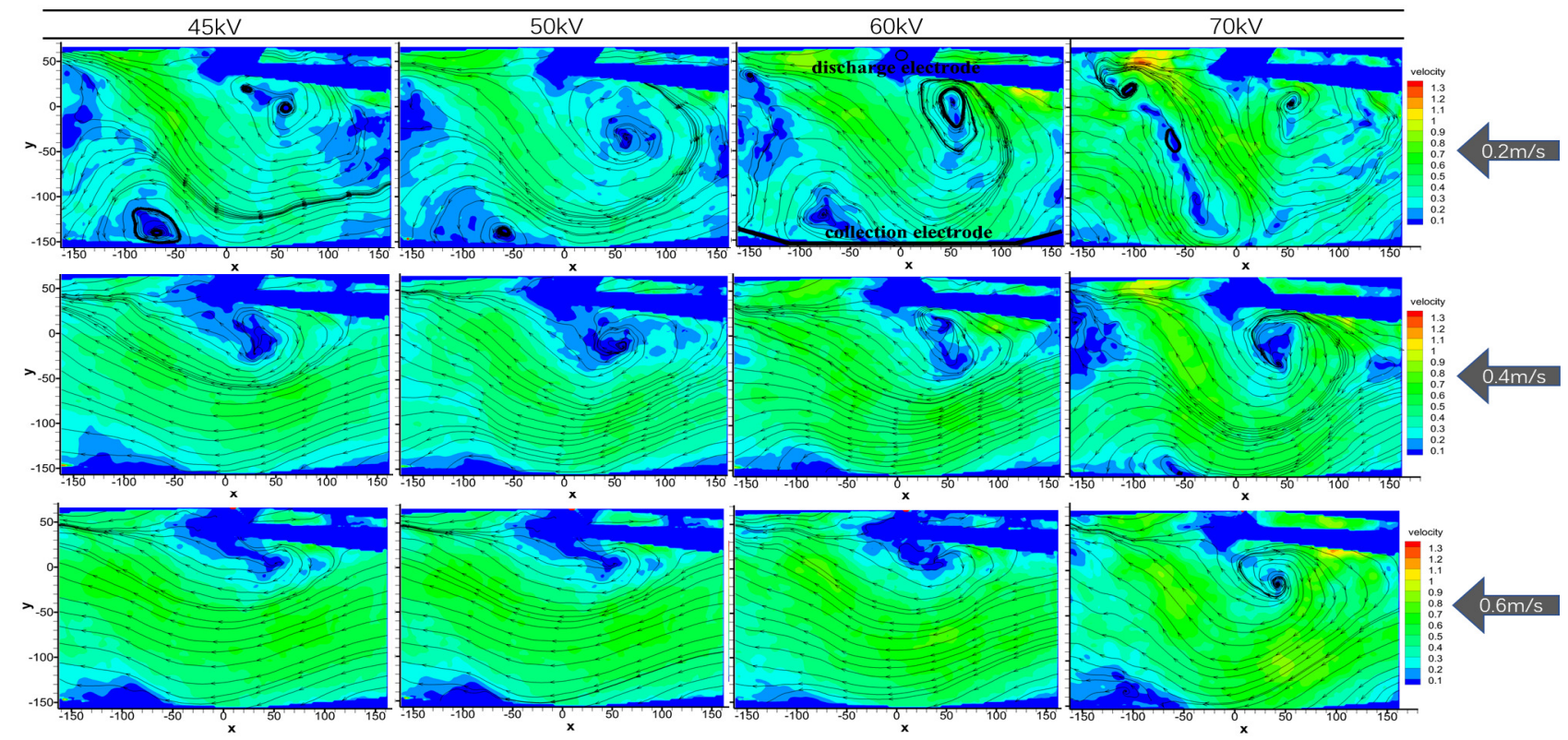

Fig.9. Streamlines with different primary flow velocity and voltage in plane 2 .

\section{E. EHD numerical analysis}

The movement of particles inside the electrostatic precipitator mainly depends on the balance between the electric force and the inertial forces. $\mathrm{Re}$ is the dimensionless number used to characterize the effect of viscosity. In the experiment, the ratio of the EHD value to the Reynolds number squared can be used to measure the influence of the secondary flow EHD in the electrostatic precipitator relative to the mainstream. The EHD value is calculated using the formula [21]:

$$
\begin{gathered}
E H D=I \times L^{3} /\left(A \times \rho \times v^{2} \times \mu_{i}\right) \\
R e=U_{0} L / v
\end{gathered}
$$

$\mu$ i - ion mobility $=2 \times 10^{-4}\left(\mathrm{~m}^{2} / \mathrm{Vs}\right)$;
L - characteristic length (the distance between plate electrodes) $=400 \mathrm{~mm}$;

$v$ - air kinematic viscosity $=1.57 \times 10^{-5} \mathrm{~m} / \mathrm{s}$;

I - represent discharge current;

The effect of increase in voltage and primary flow velocity on $\mathrm{EHD} / \mathrm{Re}^{2}$ value is shown in Table 1. As the $\mathrm{EHD} / \mathrm{Re}^{2}$ value increases, the role of the secondary flow relative to the primary flow becomes more and more evident. When $\mathrm{U}_{0}$ is $0.2 \mathrm{~m} / \mathrm{s}$ and $0.4 \mathrm{~m} / \mathrm{s}$, it can be seen that the values are all greater than 1 , indicating that the effect of the EHD is greater than that of the mainstream at this time, especially at $0.2 \mathrm{~m} / \mathrm{s}$, where the EHD force is completely dominant. When $\mathrm{U}_{0}$ is $0.6 \mathrm{~m} / \mathrm{s}$ and the voltage is $45 \mathrm{kV}$ and $50 \mathrm{kV}$, the $\mathrm{EHD} / \mathrm{Re}^{2}$ value is less than 1 . That of $70 \mathrm{kV}$ is only similar to that of $50 \mathrm{kV}$ at $0.4 \mathrm{~m} / \mathrm{s}$, which is consistent with the influence of the primary flow velocity seen on the streamline diagram on the secondary flow. 
Table $1 . \mathrm{EHD} / \mathrm{Re}^{2}$ value for different primary flow velocity and voltage.

\begin{tabular}{ccccc}
\hline \multirow{2}{*}{$\begin{array}{c}\text { primary flow velocity } \\
(\mathrm{m} / \mathrm{s})\end{array}$} & 45 & 50 & 60 & 70 \\
\cline { 2 - 5 } & 6.34 & 7.35 & 11.31 & 15.42 \\
\hline 0.2 & 1.58 & 1.84 & 2.83 & 3.85 \\
0.4 & 0.70 & 0.82 & 1.26 & 1.71 \\
\hline
\end{tabular}

\section{F. Three-dimensional analysis}

The $3 \mathrm{D}$ view of the streamline diagram of $70 \mathrm{kV}$ in plane 1-3 is shown in Fig.10. Since $V_{Z}$ (velocity in Z-direction) is not apparent when the voltage is small, this paper only analyzes the situation when the voltage is $70 \mathrm{kV}$. The position of the spike tubular electrode and the specific orientation of the spike tip are indicated. Due to the influence of the EHD flow, the airflow creates a highintensity disturbance in the three-dimensional space, but there are certain rules for these disturbances. In correspondence with the two-dimensional streamline diagram, four three-dimensional vortices can be seen in plane 3, as shown in Fig.10., while plane 1 forms a relatively strong disturbance, especially in the tip end region along the flow.
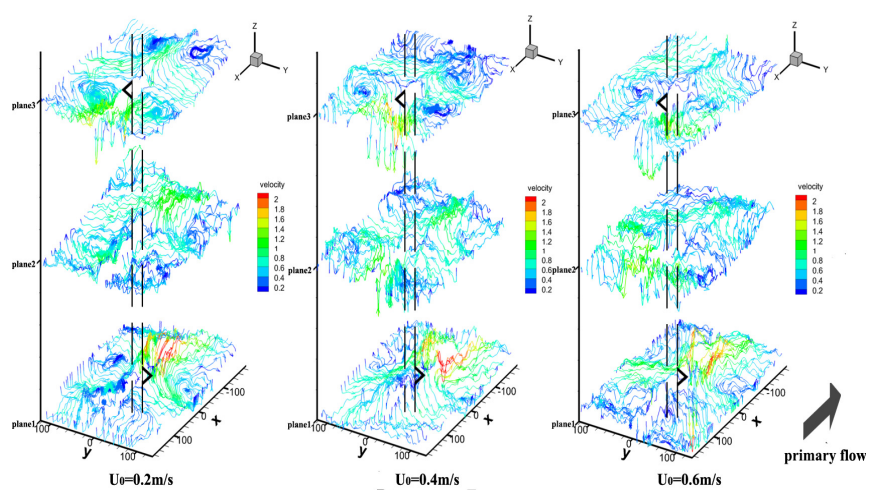

Fig.10. 3D view of flow streamlines of $70 \mathrm{kV}$ at different planes.

A comparison of $\mathrm{V}_{\mathrm{Z}}$ distribution and 2D streamline diagram in plane 1 is shown in Fig. 11. The left column of this figure is the velocity $\mathrm{V}_{Z}$ distribution and the right column is the corresponding $2 \mathrm{D}$ streamline diagram. It should be noted that due to the shooting angle, a negative value of $\mathrm{V}_{\mathrm{Z}}$ indicates that the actual velocity is upward, and a positive value of $\mathrm{V}_{\mathrm{Z}}$ indicates it is downward. In plane 1, there is an obvious high-speed jet in a downward direction at the tip of the spike. When the primary flow velocity is $0.2 \mathrm{~m} / \mathrm{s}$, $0.4 \mathrm{~m} / \mathrm{s}$, and $0.6 \mathrm{~m} / \mathrm{s}, \mathrm{V}_{\mathrm{z}}$ of this high-speed jet region can reach $0.9 \mathrm{~m} / \mathrm{s}, 1 \mathrm{~m} / \mathrm{s}, 1.2 \mathrm{~m} / \mathrm{s}$, respectively. Different from the tip of the spike, the $\mathrm{V}_{\mathrm{Z}}$ of the whole vortex region (including the vortex center and the area near the plate) is upward owing to the strong EHD effect. Comparing the two kinds of images, we can see that the vortex region and the $V_{Z}$ of the upward region are highly consistent. With the increase of primary flow velocity, the scope of influence of this "ascending vortex" is gradually reduced. When the primary flow velocity is $0.2 \mathrm{~m} / \mathrm{s}, 0.4 \mathrm{~m} / \mathrm{s}$, and $0.6 \mathrm{~m} / \mathrm{s}, \mathrm{Vz}$ of this "ascending vortex" can reach $-1.0 \mathrm{~m} / \mathrm{s},-0.8 \mathrm{~m} / \mathrm{s}$, $0.7 \mathrm{~m} / \mathrm{s}$, respectively. In the bending area of the C-type plate, there is an upward z-direction velocity, which is smaller, even close to $0 \mathrm{~m} / \mathrm{s}$.
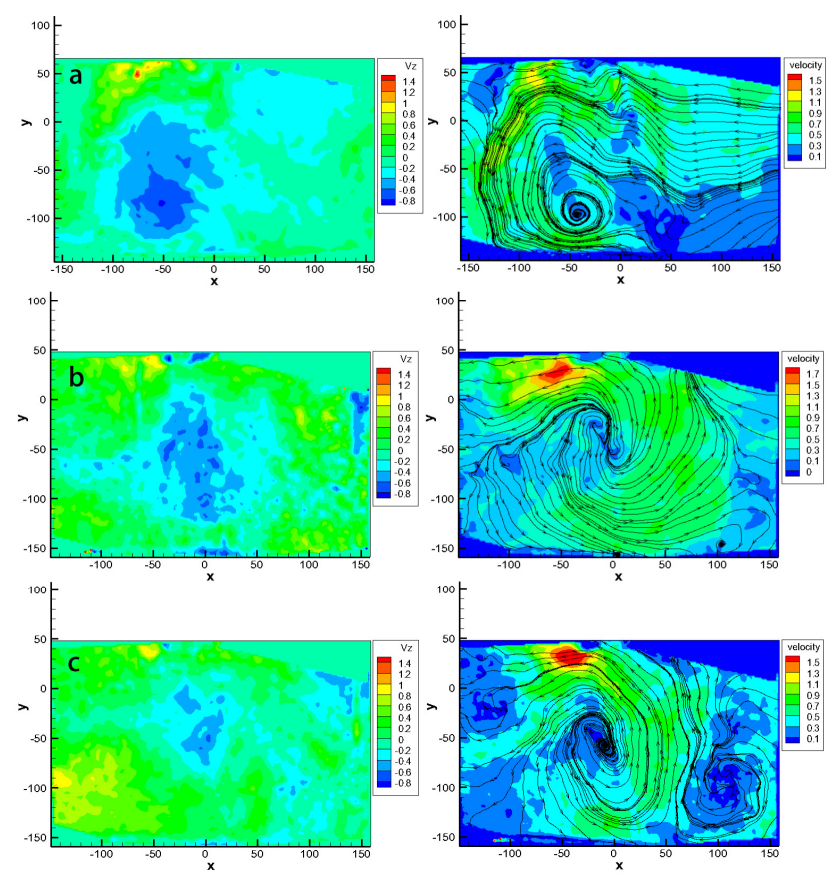

Fig.11. Comparison of $\mathrm{V}_{Z}$ distribution and two-dimensional streamline diagram of $70 \mathrm{kV}$ in plane $1\left(\mathrm{a} . \mathrm{U}_{0}=0.2 \mathrm{~m} / \mathrm{s}\right.$, b. $U_{0}=0.4 \mathrm{~m} / \mathrm{s}, \mathrm{c} . \mathrm{U}_{0}=0.6 \mathrm{~m} / \mathrm{s}$ )

In plane 2 , there are also obvious vortices and high-speed jet regions. However, due to the influence of the flow field of the other two planes, the three-dimensional flow pattern is chaotic and irregular.

A comparison of $\mathrm{V}_{\mathrm{Z}}$ distribution and two-dimensional streamline diagram in plane 3 is shown in Fig.12. In plane 3 , although the tip of the spike also produces a highvelocity jet, the jet velocity is significantly lower than that in plane 1 and it can be seen that the "ascending vortex" still exists, though its region scope is reduced, and the size of the upward $\mathrm{V}_{\mathrm{Z}}$ is increased. With all the three primary flow velocities $\left(\mathrm{U}_{0}=0.2 \mathrm{~m} / \mathrm{s}, \mathrm{U}_{0}=0.4 \mathrm{~m} / \mathrm{s}, \mathrm{U}_{0}=0.6 \mathrm{~m} / \mathrm{s}\right)$, $\mathrm{V}_{\mathrm{Z}}$ can far exceed $-1 \mathrm{~m} / \mathrm{s}$. This means that in plane 3 , a concentrated high-speed rising vortex is formed. It should be noted that there are also upward vortices downstream of 
the gas flow near the plate. This flow may cause the particles deposited at the plate to be entrained again and moved upward, affecting the collection of particles on other planes.
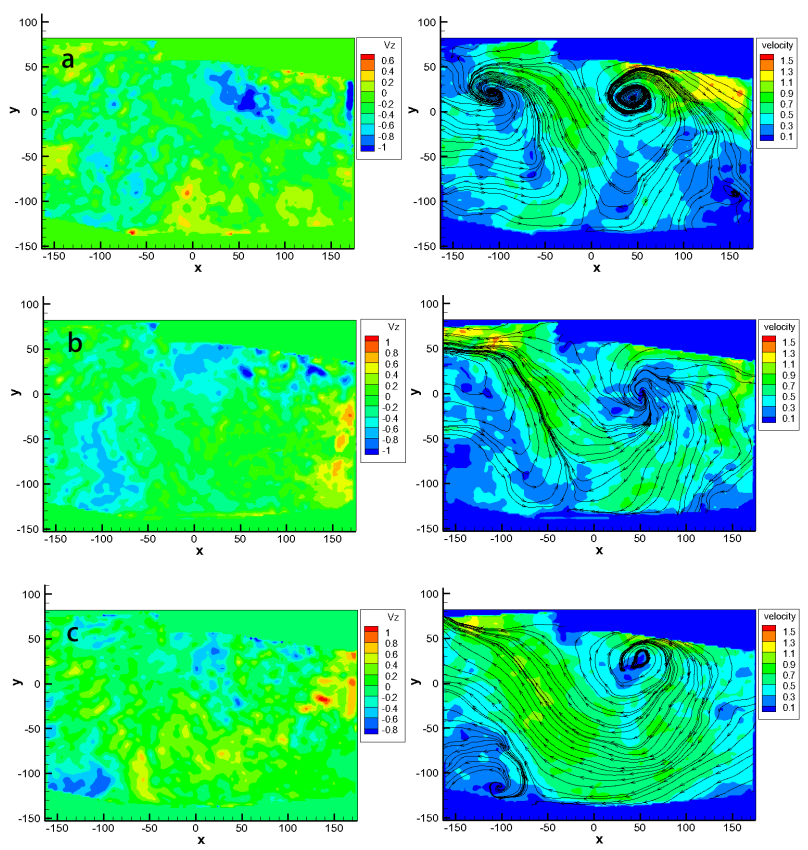

Fig.12. Comparison of $\mathrm{V}_{Z}$ distribution and two-dimensional streamline diagram of $70 \mathrm{kV}$ in plane 3 (a. $\mathrm{U}_{0}=0.2 \mathrm{~m} / \mathrm{s}$, b. $U_{0}=0.4 \mathrm{~m} / \mathrm{s}$, c. $U_{0}=0.6 \mathrm{~m} / \mathrm{s}$ ).

\section{CONCLUSIONS}

The following conclusions are drawn in this study, after observing and analyzing the EHD flow of spiked tubular electrode corona discharge in a wide electrostatic precipitator $(400 \mathrm{~mm})$ :

1) The flow field in the wide type electrostatic precipitator is symmetrical around the section of spiked tubular electrode symmetry. High speed jet will be produced at the tip of the spike under corona discharge and the air flow in the ESP will form a vortex motion due to EHD. When the tip of the spike is oriented in the direction of primary airflow, a counter-clockwise vortex is formed. When the tip of the spike is in the opposite direction of the primary airflow, a clockwise vortex is formed and the flow velocity around the vortex is high, and by contrast, a low flow velocity region appears in the center of the vortex. The vortex can affect the velocity near the plate electrode. When the spike tip is directed to the primary flow, the flow velocity near the plate will decrease with the increase of the primary flow velocity. However, when spike tip is in the opposite direction of the primary flow, the flow velocity near the plate will increase with the increase of the primary flow velocity, which is caused by the different rotation direction of the vortex.

2) When the voltage is $60 \mathrm{kV}$ and $70 \mathrm{kV}$, at three primary flow velocities, the values of the EHD/ $\mathrm{Re}^{2}$ are all greater than 1. The maximum value of $\mathrm{EHD} / \mathrm{Re}^{2}$ is 15.42 .
In such conditions, the influence of the EHD flow on particle collection is dominant, and the flow field shows a strong turbulence model.

3) Through the comparison of a two-dimensional streamline and a three-dimensional streamline, it can be found that the vortex region and $\mathrm{V}_{\mathrm{Z}}$ upward region are highly consistent. The main factors affecting the "ascending vortex" are voltage, primary flow velocity, and the orientation of the tip. If the voltage exceeds a certain value, "ascending vortex" will form, otherwise, the vortex will not show obvious three-dimensional characteristics. When the spike tip of the plane is oriented in the direction of the primary flow, a large scale "ascending vortex" is formed. However, as the spike tip of the plane is opposite to the primary flow, a small scale and relatively concentrated high-speed rising vortex forms. With the increase of primary flow velocity, the influence range of this "ascending vortex" will gradually decrease, which is due to the weakening of the primary flow on the Zdirection effect of the EHD flow.

\section{ACKNOWLEDGMENT}

The researchers gratefully acknowledge the financial support of the National Key Research and Development Program of China (Grant No. 2018YFC0705300) and Natural Science Foundation of China (51408455).

\section{REFERENCES}

[1] Mizuno, A. (2000). Electrostatic precipitation. IEEE Transactions on Dielectrics and Electrical Insulation, 7 (5), 615-624.

[2] Mizeraczyk, J., Niewulis, A., Podlinski, J. (2009). Electrohydrodynamic flow and particle collection efficiency of a spike-plate type electrostatic precipitator. Journal of Electrostatics, 67 (2-3), 99104.

[3] Niewulis, A., Podlinski, J., Kocik, M., Barbucha, R. Mizeraczyk, J., Mizuno, A. (2007). EHD flow measured by 3D PIV in a narrow electrostatic precipitator with longitudinal-to-flow wire electrode and smooth or flocking grounded plane electrode. Journal of Electrostatics, 65 (12), 728-734.

[4] Anagnostopoulos, J., Bergeles, G. (2002). Corona discharge simulation in wire-duct electrostatic precipitator. Journal of Electrostatics, 54 (2), 129-147.

[5] Yamamoto, T. Velkoff, H.R. (1981). Electrohydrodynamics in an electrostatic precipitator. Journal of Fluid Mechanics, 108, 1-18.

[6] Yabe, A., Mori, Y., Hijikata, K. (2012). EHD study of the corona wind between wire and plate electrodes. AIAA Journal, 16, 340-345.

[7] Brocilo, D., Chang, J.S., Godard, R., Berezin, A.A. (1999). Numerical modelling of wire-pipe electrostatic precipitator for control of fine particulates. Journal of Aerosol Science, 30 (suppl. 1), S855-S856. 
[8] Atten, P., McCuskey, F.M.J., Lahjomri, A.C. (1987). The electrohydrodynamic origin of turbulence in electrostatic precipitators. IEEE Transactions on Industry Applications, 24 (4), 705-711.

[9] Podlinski, J., Mizeraczyk, J. (2011), Visualization of dust collection in DC-Corona-Driven electrostatic precipitator. IEEE Transactions on Plasma Science, 39 (11), 2260-2261.

[10] Mizeraczyk, J., Kocik, M., Dekowski, J., Dors, M., Podlinski, J. (2001). Measurements of the velocity field of the flue gas flow in an electrostatic precipitator model using PIV method. Journal of Electrostatics, 51 (1), 272-277.

[11] Podlinski, J., Niewulis, A., Mizeraczyk, J., Atten, P. (2008). ESP performance for various dust densities. Journal of Electrostatics, 66 (5-6), 246-253.

[12] Mizeraczyk, J., Dekowski, J., Podlinski, J., Kocik, M., Ohkubo, T., Kanazawa, S. (2003). Laser flow visualization and velocity fields by particle image velocimetry in electrostatic precipitator model. Journal of Visualization, 6 (2), 125-133.

[13] Podlinski, J., Dekowski, J., Mizeraczyk, J., Brocilo, D., Urashima, K., Chang, J.S. (2006). EHD flow in a wide electrode spacing spike-plate electrostatic precipitator under positive polarity. Journal of Electrostatics, 64 (7-9), 498-505.

[14] Podlinski, J., Niewulis, A., Mizeraczyk, J. (2009). Electrohydrodynamic flow and particle collection efficiency of a spike-plate type electrostatic precipitator. Journal of Electrostatics, 67 (2-3), 99104.
[15] Berendt, A., Mizeraczyk, J. (2016). Transition of the electrohydrodynamic two-phase flow into the singlephase flow in a needle-to-plate negative corona discharge in the finite-volume chamber. Journal of Electrostatics, 84, 90-96.

[16] Miyashita, H., Ehara, Y., Inui, T., Aoki, Y. (2018). Particle behavior analysis in a hole-type electrostatic precipitator using PIV. IEEE Transactions on Industry Applications, 54 (5), 4857-4863.

[17] Ning, Z., Podlinski, J., Shen, X., Li, S., Wang, S., Han, P., Yan, K. (2016). Electrode geometry optimization in wire-plate electrostatic precipitator and its impact on collection efficiency. Journal of Electrostatics, 80, 76-84.

[18] Ning, Z., Cheng, L., Shen, X., Li, S., Yan, K. (2016). Electrode configurations inside an electrostatic precipitator and their impact on collection efficiency and flow pattern. The European Physical Journal D, $70(6), 126$

[19] Wang, C., Xie, Z., Xu, B., Li, J., Zhou, X. (2016). Experimental study on EHD flow transition in a small scale wire-plate ESP. Measurement Science Review, 16 (3), 134-141.

[20] Podlinski, J., Berendt, A., Mizeraczyk, J. (2013). Electrohydrodynamic secondary flow and particle collection efficiency in spike-plate multi-electrode electrostatic precipitator. IEEE Transactions on Dielectrics \& Electrical Insulation, 20 (5), 1481-1488.

[21] Soldati, A. (2000). The effects of electrohydrodynamic flows and turbulence on aerosol transport and collection in wire-plate electrostatic precipitators. Journal of Aerosol Science, 31 (3), 293305.

Received May 17, 2020

Accepted August 10, 2020 